\title{
A Network Fault Isolation Method Based On Immune Theory
}

\author{
Chen Zhu*
}

Computer College, Chongqing College of Electronic Engineering; Chongqing 401331, China

\begin{abstract}
An algorithm for constrained flooding based on negative selection is proposed due to improve delay of flooding and quality of operation when the network transmit the fault information. According to the network environment different demand between delay of flooding and quality of operation, cost function of flooding delay and operation losing are set, the optimizing objective function synthesize two factor is also set, and negative selection algorithm is used to compute the area of constrained flooding. The experimental result shows that this algorithm can improve the capability in network.
\end{abstract}

Keywords: Constrained flooding, Fault isolation, Negative selection, Network invulnerability.

\section{INTRODUCTION}

With the increasing network scale and its gradually complicated structure, the complex network not only brings great convenience to the daily production and life in human society, but also sets stricter demands on the stability of the network system. In such fields as the national defense, finance and public communication, the stability of the computer network will have a huge impact on the country, and even to the security, economy and politics in the world, as a result, the stability of network has always been the emphasis and difficulties in the study on the network survivability.

When the network system should update the state or some devices go wrong, large-scale flooding should be carried out for the state or fault information of the network, and corresponding survivability enhancing technique should be taken for the fault. In the traditional OSPF Agreement [1], when the network breaks down, the network node detecting the fault will send the fault information to other nodes in the network, and update its routing table according to the routing information it grasps. Although this method can guarantee that the network will always run away from the route in the fault link, it is slow in rate of convergence and inapplicable for the real-time business. Furthermore, the flooding failure information throughout the network may lead to the oscillation in the network. The rapid convergence method [2] adopted by aiming at the slow convergence rate in the traditional agreement will also lead to the network oscillation and waste of the bandwidth resource. Though the active rerouting technology [3-5] can solve the slow convergence rate of the network, it only guarantees the normal operation for a short time, without solving the stability of the network fundamentally.

There are many reasons for the network instability, which is mainly caused failure of treating the fault timey or over-strong fault handling reaction. When fault occurs, the network system will announce the fault information across the network in large scale, which will not only increase the load of the link, but also lead to the phenomenon of network oscillation owning to the frequent fault information processing by the nodes, thus influence the stability of network. It could be seen that, the effect isolation processing for the network fault will minimize the influence on the stability of the network, and it is necessary to conduct the studies on the fault isolation technique.

In the second part, the current research status and theoretical basis of the fault isolation technique is introduced simply. In the third part, a limiting flooding method based on negative selection is proposed. In the fourth part, computer simulation and performance analysis are carried out for this method. In the end, the conclusion and direction of the follow-up study are pointed out.

\section{RELATED THEORY ABOUT FAULT ISOLATION}

\subsection{Relationship Between The Fault Isolation And Prin- ciples Of Immunology}

During the natural immune process, when the external antigen invades, the first line of defense, namely the skin and mucous membrane will resist the invasion and oppose to the antigen's damage to the living body physically. The second line of defense, including the bactericidal substance in the body fluid and phagocyte, will destroy and resist the antigen. Generally, the first two lines of defense in the immune system can prevent most antigens from invading, the third line of defense will be adopted by the immune system after the first two lines of defense became invalid, and it is the specific immunity, which is the antibody or lysozyme produced by the immune cells to eliminate the antigens that cannot be resisted by the first two lines of defense.

It is not difficult to see from the nature immune process that when the living body is invaded by the antigen, the first reaction of the immune system is to isolate the antigen out of 


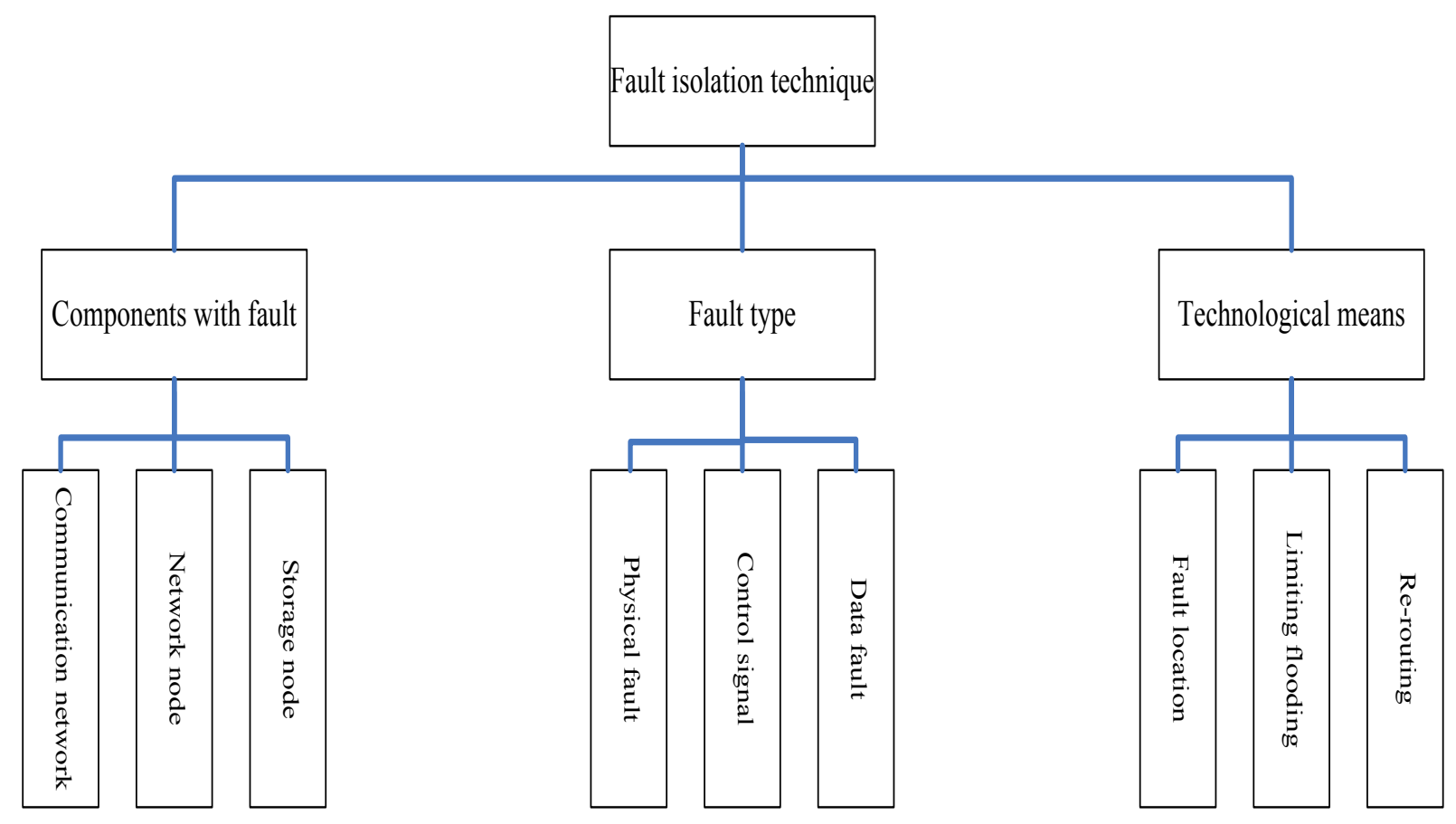

Fig. (1). Classification of fault isolation technology.

the living body or control the antigen's invasion within certain limits and wait for the specific immunity to eliminate the antigen. Such treatment to antigen can not only save the resources better, but also control the antigen to prevent its influence on the living body from spreading to the whole body.

When combining with the network survivability, once the network fails, the fault information flooding shall be conducted within the whole network range and corresponding re-routing technology shall be adopted, which will certainly lead to the waste of network computing resources and the oscillation of state. According to the studies, most network failure lasts for a short time, and most of them can be processed locally. There is no need to deluge the fault information to the full network, which is similar to the process of restricting and isolating the antigen in the immune system. The fault isolation learns from the principle of immunology, and it can realize the saving of network bandwidth resource and the stability of the network system.

\subsection{Techniques Of Fault Isolation}

There are different types of fault isolation technique from different perspectives. From the perspective of failure component [6], it can be divided into communication network fault isolation, network node fault isolation and storage node fault isolation; from the perspective of failure type [7], it can be divided into physical fault isolation, control signal fault isolation and data fault isolation; from the fault isolation technology, it can be divided into fault location technique, limiting flooding technique and re-routing technique (Fig. 1).

The fault location technology [8], namely the measures making use of the event/alarm relevance, refers to the technology of inferring the fault location and reasons by analyzing the events or alarms observed. It can pinpoint the fault location and type accurately according to the alarm condition of the network system to provide information for the follow-up fault isolation and recovery.

Limiting flooding technique [9-11], is a method taking advantage of the restrictions on the flooding range of the fault information or on the capacity of the fault information, and it can reduce the network oscillation and the waste of the bandwidth resource caused by the large-scale flooding of fault information. It can control the influence on the whole network within a certain range effectively and guarantee the stability of the network, which is a significant constituent part in the enhancement technique of the network survivability.

The re-routing technique [2-5], refers to the process in which the network makes use of the methods, such as the rapid convergence, multi-path backups or multi-topology to recover the faults in the network. It can recover the fault and re-build the route automatically within a short time and guarantee the normal operation of the network.

The fault location technique and re-routing technique are relatively mature studies. In this paper, it lays emphasis on the limiting flooding technique and a negative selectionbased limiting flooding algorithm has been put forward.

\section{NEGATIVE SELECTION-BASED LIMITING FLOODING TECHNIQUE}

The traditional limiting flooding algorithm tries to shorten the flooding delay or guarantee the business service quality. However, if attention is paid to decrease the delay, please try to limit the flooding range of the link state advertisement 
(LSA) as much as possible. During the process of shortening the delay, it will lead to the business loss for some nodes may not update the routing table in time. If it pays attention to guarantee the business service quality, it must guarantee that the network nodes can update the routing table, thus it may not lead to the loss of business. However, the large scale flooding will lead to the increase in delay and the decrease of network stability.

Therefore, it is quite necessary to propose a comprehensive limiting flooding technology considering the time delay and service quality, and the optimized objective function for the limiting flooding technique has been put forward as follow.

\subsection{Optimized Objective Function}

According to the facts, the demands raised by different external environment and different bearer service are different. Therefore, it must balance the delay and service quality comprehensively according to the different environments, and seek for relatively optimal flooding range. In this paper, the network topology stated in this paper can be described as a picture $\boldsymbol{G}=(\boldsymbol{V}, \boldsymbol{E})$, in which $\boldsymbol{V}=\left\{v_{1}, v_{2}, \cdots, v_{n}\right\}$ can be described as a peak set, and each peak corresponds to one node in the topology, $\boldsymbol{E}=\left\{e_{1}, e_{2}, \cdots, e_{m}\right\} \subseteq \boldsymbol{V} \times \boldsymbol{V}$ stands for the set of edges in the picture, and each edge corresponds to the link in the topology structure. $n=|\boldsymbol{V}|$ stands for the number of nodes, $m=|\boldsymbol{E}|$ stands for the number of nodes. The variances needed in the optimized objective function are defined as follows:

Suppose, after the node $r$ detects the link failure, the set made by flooded nodes will be $\boldsymbol{V}_{\text {flood }}(r)$, and $\boldsymbol{V}_{\text {flood }} \subseteq \boldsymbol{V}$.

\subsubsection{Flooding Delay Cost}

In the communication network, there is a close connection between the flooding delay and network link cost. As a result, the link cost is used to represent the flooding delay cost, and the flooding delay cost of the flooding set $\boldsymbol{V}_{\text {flood }}(r)$ can be described as:

$$
\begin{aligned}
& T(r)=\frac{\max \left[\text { length }\left(\operatorname{spt}\left(r, v_{i}\right)\right)\right]-\min \left[\text { length }\left(\operatorname{spt}_{b}(r)\right)\right]}{\max \left[\operatorname{length}\left(\operatorname{spt}_{b}(r)\right)\right]-\min \left[\text { length }\left(\operatorname{spt}_{b}(r)\right)\right]}, \\
& v_{i} \in V_{\text {flood }}(r)
\end{aligned}
$$

In which, $\operatorname{spt}_{b}(r)$ stands for some branch of the shortest path tree generated by taking $r$ as the root node, $\operatorname{spt}\left(r, v_{i}\right)$ stands for the path from node $r$ to node $v_{i}$ among the shortest path tree which regards the $r$ as the root node. In equation (1), the largest path cost within the flooding range is uniformized to describe the flooding delay cost.

\subsubsection{Flooding Business Loss}

In the traditional flooding agreement, when a link failure is detected, LSA will send it to all the routers within the range. Thus it can guarantee that all the routers can update the routing information in time, and guarantee the normal operation of the bearer service. However, the flooding within the whole network may lead to the increase in flooding delay and the decrease of the network stability. Therefore, the limiting flooding technique is proposed. In the limiting flooding, LSA can only be sent to the routers within certain range. On the other hand, the small flooding range may lead to the failure in the timely advertisement of the link information, and as a result, the routers out of the flooding range will still use the original routing table and lead to the business loss.

The business loss is described as:

$$
\begin{aligned}
& R_{\text {loss }}(r)=\frac{\sum_{v_{n}=1}^{n-V_{n} \mid} \sum_{v=1}^{n-1} \operatorname{path}\left(v_{n}-v\right)}{\left(n-\left|V_{n}\right|\right)(n-1)}, \\
& v_{n} \notin \boldsymbol{V}_{\text {flood }}(r)
\end{aligned}
$$

It represents the business loss caused by the use of flooding set $\boldsymbol{V}_{\text {flood }}(r)$ after the link failure is detected by node $r$, path $\left(v_{n}-v\right)$ stands for if the routing table used in the path $v_{n}-v$ currently is smooth or not. If it is smooth, it will be 0 , or it will be 1 , and $v_{n}$ is any node out of the flooding set, whiles $v$ is any node except the node $v_{n}, V_{n}$ is a set constituted by all the $v_{n}$.

\subsubsection{Objective Function}

Since the set limiting flooding range may influence factors in two aspects, namely the flooding delay and business loss, it must consider the flooding delay and business loss according to the bearer service of the network environment. Therefore, the optimized target function considering the two aspects of factors can be defined as:

$$
F_{\text {flood }}(r)=\alpha T(r)+(1-\alpha) R_{\text {loss }}(r)
$$

In the equation, $F_{\text {flood }}(r)$ is the cost paid to the flooding LSA of the flooding set $\boldsymbol{V}_{\text {flood }}(r)$ by the originating node $r$. $\alpha$ is the impact factor of delay and $\alpha \in[0,1]$, suggesting the attention of network environment and network bearer service on the influence of flooding delay. The greater $\alpha$ is, the more attention it will paid to influence of the network delay.

It can be seen from equation (1) to (3) that the flooding range has a direct influence on the performance of the network. In order to reach optimum among various performance indexes, the artificial intelligent algorithm can be adopted to seek for the optimum solution by setting the objective 
function. In this paper, the negative selection algorithm is combined to put forward a limiting flooding algorithm based on the negative selection.

\subsection{Limiting Flooding Algorithm}

Suppose that the surveyed area is $20 \mathrm{~m} \times 20 \mathrm{~m}$ square, and it is divided into $20 \times 20$ grid points with the same.

The traditional traversal optimization technique may not find out the optimal solution owning to its poor directivity. As a result, the intelligent algorithm should be found out when determining the limiting flooding range.

Definition 1, the vector $\boldsymbol{N}_{\text {flood }}(r)$ is defined as the node determinant to show if the node exists in the flooding range that takes $r$ as the node, namely the characteristics of this flooding range, $\boldsymbol{N}_{\text {flood }}(r)$ is represented as:

$$
\boldsymbol{N}_{\text {flood }}(r)=\left[n f_{1}, n f_{2}, \cdots, n f_{n}\right]
$$

In this equation, if node $i$ exists in the flooding range, the corresponding $n f_{i}$ is 1 , or it is 0 . The negative selection algorithm can recognize the self and nonself effectively for generating the nonself detector, which will select the appropriate individuals for the immune system effectively. It has been widely applied in the artificial intelligence field. The principle of the negative selection algorithm will be introduced briefly as follows.

\subsubsection{Negative Selection Algorithm}

In the biological immune system, there is a negative selection mechanism for preventing the extreme reaction of the immune cells and avoiding the immune cell's injury to the self. Such a mechanism can not only delete the cells killing the self, but also keep and detect the nonself cells. Such detection and selection model is called as the negative selection algorithm [12].

Suppose the characteristics of the immune cells and self cells can be represented by the determinant, which can be represented by corresponding character string in artificial intelligence. Provide that $P_{M}$ is the probability of the mutual match between two groups of character string in specific matching rule, then the probability of a group of character string not matching the self is $1-P_{M}$, when the self set consists of $N_{S}$ independent elements, the probability of a group of character string not matching any group of character string in the self set is $f$ :

$$
f=\left(1-P_{M}\right)^{N_{S}}
$$

The substance of the negative selection is to generate a nonself detector, which will match the character string about to be detected with the elements in the nonself detector. If they can match mutually, then the character string about to be detected will be listed as the threatening character string.
It is not difficult to conclude that the relationship between the number of initial nonself detector $N_{R_{0}}$ and tem umber of mature nonself detector $N_{R}$ is:

$N_{R}=N_{R_{0}} \times f$

The probability of failing to detect the self changes by the detector set with $N_{R}$ mature nonself detector is:

$P_{f}=\left(1-P_{M}\right)^{N_{R}}$

It can be learnt from equation (6) and (7) that:

$N_{R}=\frac{\ln P_{f}}{\ln \left(1-P_{M}\right)}$

$N_{R_{0}}=\frac{\ln P_{f}}{\left(1-P_{M}\right)^{N_{S}} \ln \left(1-P_{M}\right)}$

Therefore, the number of initial nonself detector needed for generating a mature nonself detector set can be figured out. The flow of negative selection algorithm is:

Define the self set $S$ and related parameters, and the character string about to be generated randomly will match the self set. If they match to each other, this character string shall be deleted, if not, this character sting will be added to the mature nonself detector set $R$. In the end, $R$ and $S$ will be compared to monitor the invasion of the external antigen. Under such mechanism, as long as the definition of the self set is complete, there won't be conditions in which the self is recognized as the nonself generally.

\subsubsection{Settings for Self Set}

Combining with the limiting flooding technique, the settings of self set should consider the general conditions of the flooding set, and those individuals that are not qualified should be put into the self set, thus those invalid individuals will not occur in the nonself detector.

If the node $r$ is regarded as the source node, its limiting flooding set should be equipped with three characteristics:

Firstly, the flooding set should be connected, namely all the nodes included in the flooding set should connect to each other, or the part of LSA of the source node fails in flooding or connecting, may not be able to realize the transmission of LSA in flooding set.

Secondly, the transmission delay of the flooding set shall not be too big. The limiting flooding aims to restrict the transmission of LSA in a certain delay and scope, if the transmission delay of the flooding set is too big, it will contradict to the purpose of the limiting flooding. As a result, the delay cost of the flooding set must be controlled.

Thirdly, the business loss of the flooding set shall not be too big. If the business loss of the bearer service caused by the limiting flooding is too big, this flooding set is not applicable for limiting flooding. 
The self set can be described as:

$\operatorname{Self}(r)=\left[\operatorname{Self}_{1}(r), \operatorname{Self}_{2}(r), \cdots, \operatorname{Self}_{x}(r), \cdots\right]$

In which, the elements in the $\operatorname{Self}_{x}(r)$ is the node determinant of the xth individual in the self set Self $(r)$.

Based on the above three characteristics of the flooding set, the node determinant $N_{\text {flood }}(r)$ of each element in the self set $\operatorname{Self}(r)$ should be equipped with the following one or several features:

Firstly, the figure composed by the nodes in $N_{\text {flood }}(r)$ is not connecting.

Secondly, the flooding delay cost $T(r)$ of the figure composed by the nodes in $\boldsymbol{N}_{\text {flood }}(r)$ is greater than the delay threshold $\delta_{T}$, namely the delay of the limiting flooding exceeds the affordable range of the network.

Thirdly, the business loss $R_{\text {loss }}(r)$ of the figure composed by the nodes in $N_{\text {flood }}(r)$ is greater than the business loss threshold $\delta_{\text {loss }}$, namely the business loss caused by the limiting flooding is too big, which has a relatively large influence on the bearer service of the network..

\subsubsection{Calculation of Affinity}

The affinity is the degree of mutual matching between the elements in self set and the immune cell elements. The greater the affinity is, the closer the two will be, or vice versa.

Definition 2 The affinity of the immature detector and self set is defined as:

$A_{x, y}=\frac{1}{n} \sum_{i=1}^{n}\left(1-\left|n f_{i}(x)-n f_{i}(y)\right|\right)$

In the equation, $A_{x, y}$ stands for the affinity between the immature immune cell element $x$ and the self-set element $y$ , and it is decided by each sequence codon within the element. The more the same digit of the corresponding codon is, the greater the affinity between the two will be.

\subsubsection{Flow of the Limiting Flooding Algorithm}

The intelligent algorithm and basic settings needed by the limiting flooding are given. And a limiting flooding algorithm combining with the negative selection principle is proposed, and the specific flows are shown as follows:

(1) Suppose the flooding source node number $r=1$.

(2) Check the number $r$, if $r \leq n$, the self set of the source node is generated according to the source node $r$ and the characteristics of the self set in section 3.2.2, or please turn to the step (6).

(3) A character string about ${ }^{n}$ in length is generated randomly. Compare this character string with the elements in self set. If the affinity between the elements in self set and this character string is 1 , this character string should be deleted and a new one should be generated. If this character string does not belong to the element in the self set, then it should be added into the mature detector.

(4) Check if the number of elements in the mature detector set satisfies the set value. If not, please return to step (3), or please continue the step (5).

(5) Compare the optimized target function value $F_{\text {flood }}(r)$ of the elements in the mature detector, and select the minimum element and store it as the source node $r$ in the flooding set $\boldsymbol{V}_{\text {flood }}(r)$, and meanwhile, $r$ will increase by 1 and turn to step (2).

(6) Finish the generation of all nodes in the flooding set.

(7) When the link failure is detected by a certain node, LSA will be sent within the flooding of the node, and the algorithm ends.

With active method, the flooding range of the node will be stipulated before the failure is detected by the node in this algorithm, which saves the time for calculating the flooding range in time of failure, and the flooding range is optimized from the comprehensive consideration of reducing the flooding delay and business loss. The experimental analysis will be conducted for the performance of this algorithm.

\section{PERFORMANCE ANALYSIS}

The experiment topology adopts the US network topology composed by 14 nodes and 21 links, as shown in (Fig. 2). In general network environment, since various measures are applied by the system to guarantee the normal operation of the network, more attention should be paid to the influence of flooding delay in the study on limiting flooding technology. As a result, the delay factor $\alpha=0.7$, namely the network attaches more importance to the delay. Owning to the small scale of the experimental topology, the delay threshold $\delta_{T}$ and business loss threshold $\delta_{\text {loss }}$ is set as 1 .

\subsection{Delay Improvement}

The dijkstra algorithm is adopted to calculate the routing table of the US network topology, and the delay cost caused by flooding LSA of each node is counted by this algorithm, the algorithm stated in reference [9], and algorithm without using limiting flooding.

It can be seen from (Fig. 3) that, the main thought of the limiting flooding algorithm based on the connected dominating set is to protect the business flows as much as possible. As a result, when compared to the algorithm without limiting 


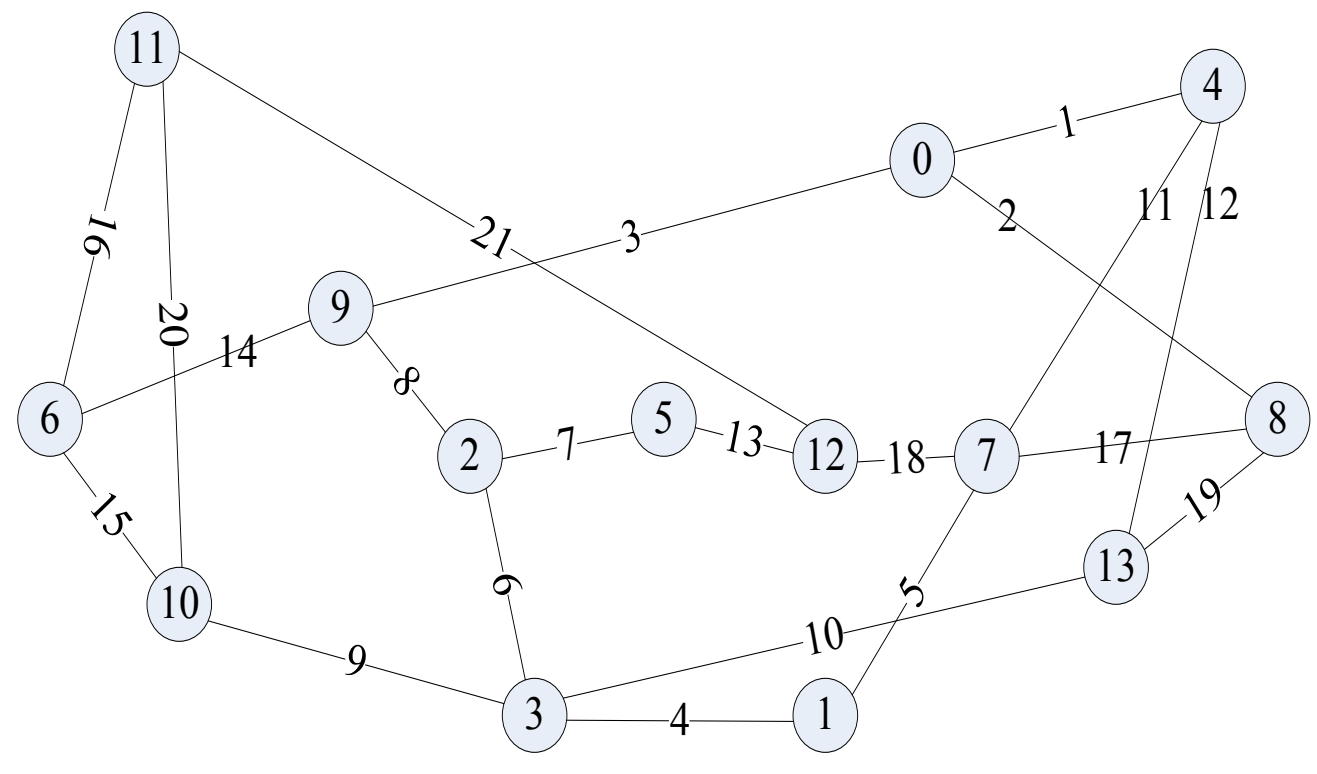

Fig. (2). The topological structure of the network.

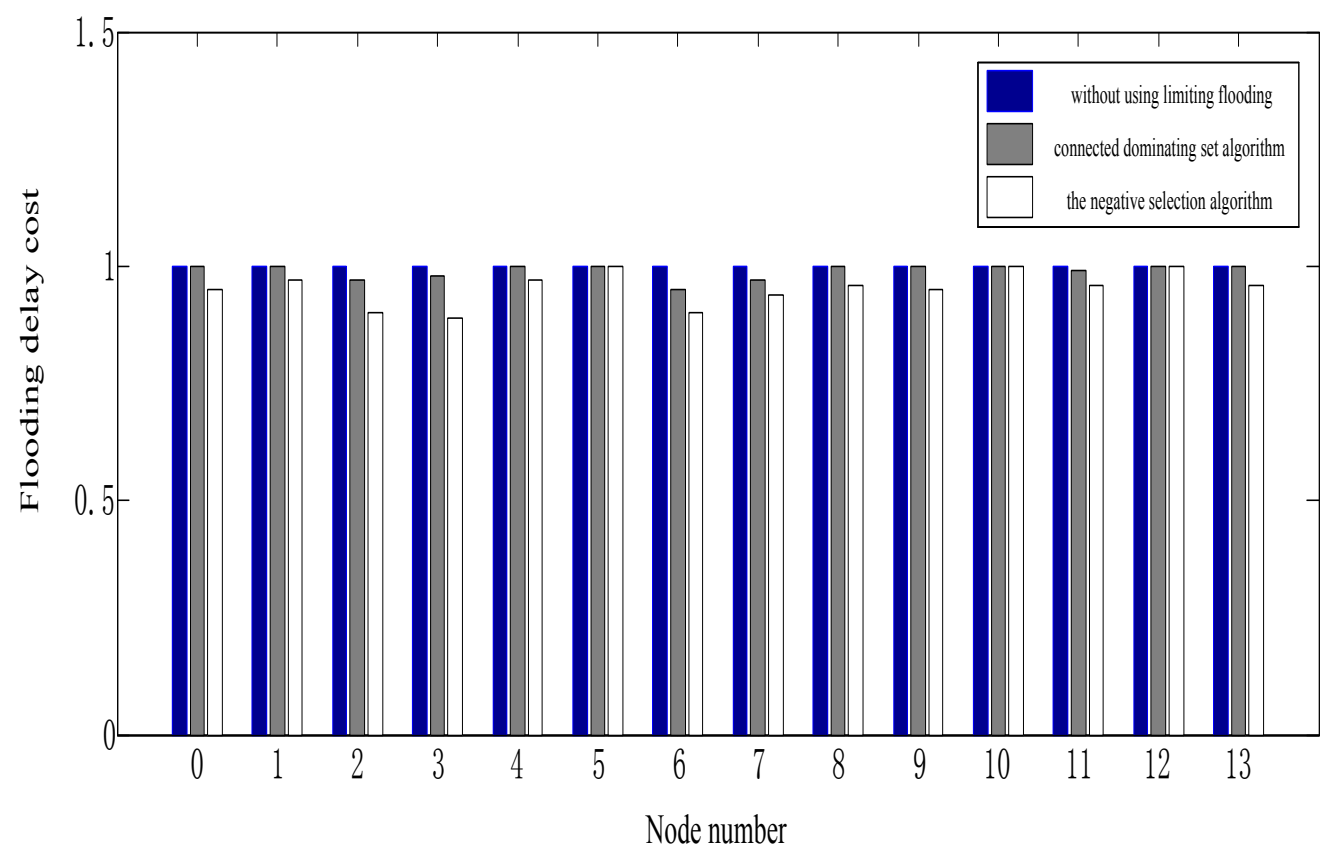

Fig. (3). Flooding delay cost.

flooding, its advantage in the improvement of reducing the delay cost is not so obvious. But in the initial setting of the experiment, it sets higher demands on the ability of reducing the delay cost by the algorithm based on the negative selection. Therefore, it can be seen that the delay cost of the algorithm based on the negative algorithm in the experiment is smaller than that of the other two algorithms, proving that the limiting flooding algorithm based on the negative selection reduces the flooding delay cost effectively.

\subsection{Business Loss Improvement}

In this experiment, the business loss caused by the flooding range of each node is counted by this algorithm, the algorithm mentioned in reference [10] and the algorithm without flooding.

The business loss cost of each node is shown in (Fig. 4). It can be seen from the figure that, the algorithm without using the limiting flooding enables all the nodes in the 


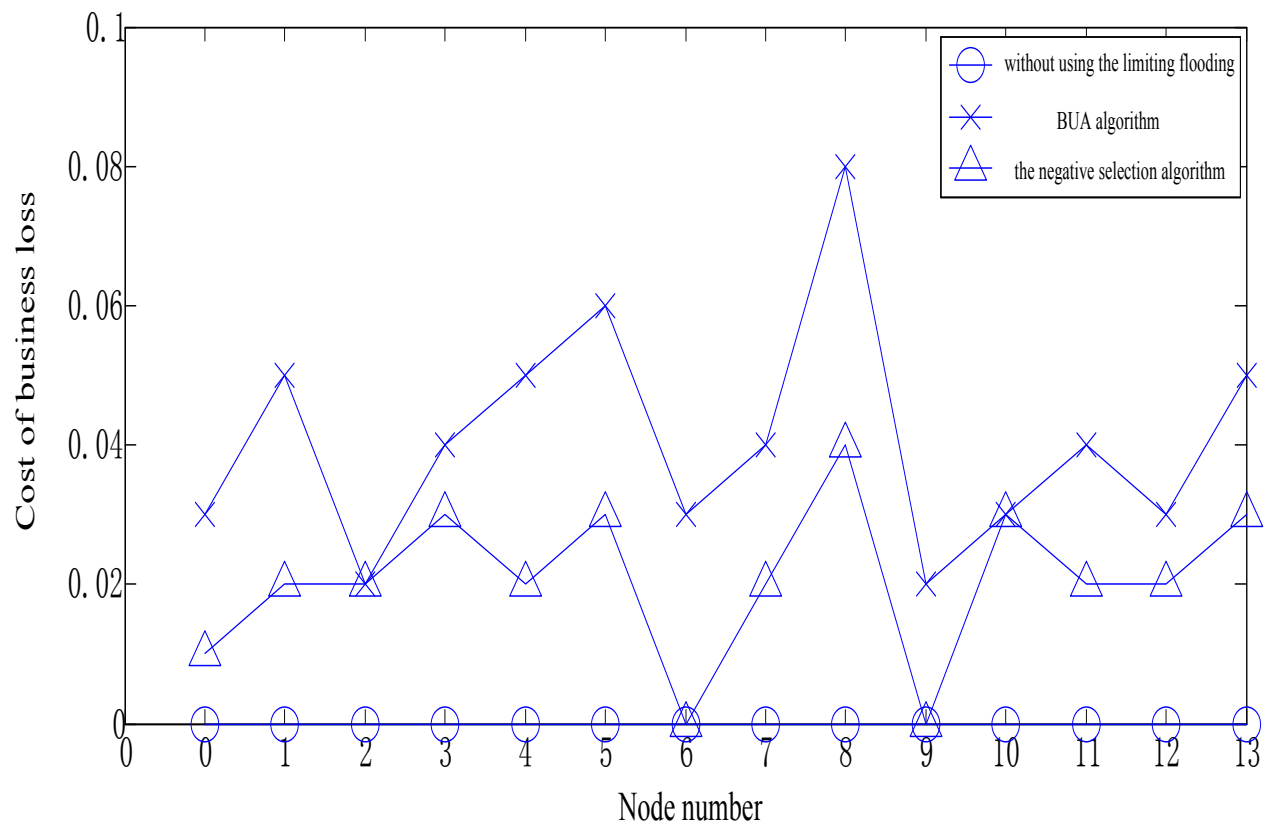

Fig. (4). Cost of business loss.

network to update the routing table after receiving the LSA, when the business loss cost of the negative selection algorithm which considers the reduction of business loss comprehensively on the basis of considering reducing the flooding delay is smaller than the BUA algorithm which only considers about the reduction of flooding delay.

It can be seen from this experiment, the limiting flooding algorithm based on the negative selection can find out the optimum solution according to the demands of the network in the aspects of reducing the flooding delay and business loss, which will guarantee the stability of the network and the quality of the service to a maximum degree.

\section{CONCLUSION}

In different environments, the network system has different demands on various network indexes. Under most circumstances, the network cannot guarantee that all its performances are in the optimum state. Therefore, in time of limiting flooding, the two aspects of factors, namely the flooding delay and business loss, shall be taken into consideration. The flooding algorithm employs the intelligent algorithm to seek for the optimum flooding range of each node under the comprehensive influence of the two aspects, thus to guarantee the stability of the network and the reliability of the transmission from the two aspects to a maximum degree. It proves that this algorithm can improve the performance of the network. In the fellow-up study, the study on the setting of each index parameter in the limiting flooding algorithm of the network should be strengthened to get the algorithm adapt to various network environments accurately.

\section{CONFLICT OF INTEREST}

The authors confirm that this article content has no conflicts of interest.

\section{ACKNOWLEDGEMENTS}

Declared none.

\section{REFERENCES}

[1] J. Moy, “OSPF Version 2” [E-book] Available: http://www.ietf.org/rfe/rfc2328.txt, 1998.

[2] C. Alaellinoglu, V. Jacobson and H. Yu, "Towards millisecond IGP convergence". [E-book] Available: http://www.nanog.org/meetings/nanog20/abstracts.php?pt=MTA3MiZuYW5vZzlw\&nm=nanog 20,2000 .

[3] X. Zhang and A. Perrig, "Correlation-resilient path selection in multi-path routing", In: Proceedings of IEEE Globecom, 2010.

[4] M. Xu, Y. Yang and Q. Li, "Selecting shorter alternate paths for tunnel-based IP fast reroute", Computer Networks, vol. 56, no. 2, pp. 845-857, 2012

[5] A. Kvalbein, A. Hansen, T. Cicic, S. Gjessing and O. Lysne "Multiple routing configurations for fast IP network recovery", IEEE/ACM Transactions on Networking, vol. 17, no. 2, pp. 473486, 2009.

[6] L. Wu, H. Luo and Z. Ai, "Proactive fault management in large scale computing systems", Journal of Huazhong University of Science and Technology, vol. 38, no. S1, pp. 20-24, 2010.

[7] S. Zhou, Z. Ou and Y. Yuan, "The technology of on-line diagnosis, fault isolation and dynamic rebuilding for network", Application Research of Computers, vol. 20, no. 1, pp. 92-93, 2003.

[8] Y. Zhang, X. Meng and Z. Li, "Analysis of the alarm correlation on the basis of SVM and simulation logic", Application Research Of Computers, vol. 28, no. 2, pp. 685-688, 2011.

[9] T. Yu, S. Chen, Z. Qin, "A rerouting scheme using connected dominating set in large-scale disaster scenario", Chinese High Technology Letters, vol. 18, no. 1, pp. 11-15, 2008. 
[10] P Narvaez, "Routing Reconfiguration in IP Networks", Massachusetts, USA: Massachusetts Institute of Technology, 2000.

[11] X. Wang, Y. Yang, M. Xu, "Low load and reliability flooding algorithm ERSN for link-state routing protocols", Application Research of Computers, vol. 25, no. 1, pp. 56-58, 2008.
[12] Y. Zhang, "Research on Negative Selection Algorithm of Artificial Immune System", Master's Thesis of Zhejiang University, 2007.

Received: November 26, 2014

Revised: January 05, 2015

Accepted: January 19, 2015

(C) Chen Zhu; Licensee Bentham Open.

This is an open access article licensed under the terms of the Creative Commons Attribution Non-Commercial License (http://creativecommons.org/licenses/by-nc/3.0/) which permits unrestricted, non-commercial use, distribution and reproduction in any medium, provided the work is properly cited. 